
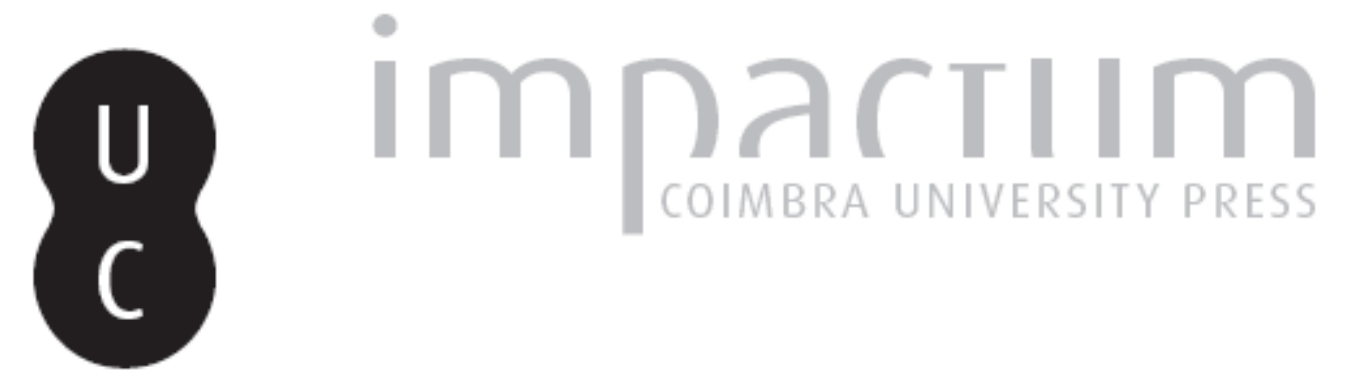
La théorie de la vision dans Timée (45B2-D2) et son commentaire par Calcidius (IVE
S. de notre ère)

Autor(es): Bakhouche, Béatrice

Publicado por: Imprensa da Universidade de Coimbra

URL persistente:

URI:http://hdl.handle.net/10316.2/42238

DOI:

DOI:https://doi.org/10.14195/2183-4105_5_3

Accessed : $\quad$ 26-Apr-2023 07:01:43

A navegação consulta e descarregamento dos títulos inseridos nas Bibliotecas Digitais UC Digitalis, UC Pombalina e UC Impactum, pressupõem a aceitação plena e sem reservas dos Termos e Condições de Uso destas Bibliotecas Digitais, disponíveis em https://digitalis.uc.pt/pt-pt/termos.

Conforme exposto nos referidos Termos e Condições de Uso, o descarregamento de títulos de acesso restrito requer uma licença válida de autorização devendo o utilizador aceder ao(s) documento(s) a partir de um endereço de IP da instituição detentora da supramencionada licença.

Ao utilizador é apenas permitido o descarregamento para uso pessoal, pelo que o emprego do(s) título(s) descarregado(s) para outro fim, designadamente comercial, carece de autorização do respetivo autor ou editor da obra.

Na medida em que todas as obras da UC Digitalis se encontram protegidas pelo Código do Direito de Autor e Direitos Conexos e demais legislação aplicável, toda a cópia, parcial ou total, deste documento, nos casos em que é legalmente admitida, deverá conter ou fazer-se acompanhar por este aviso.

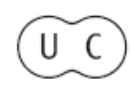




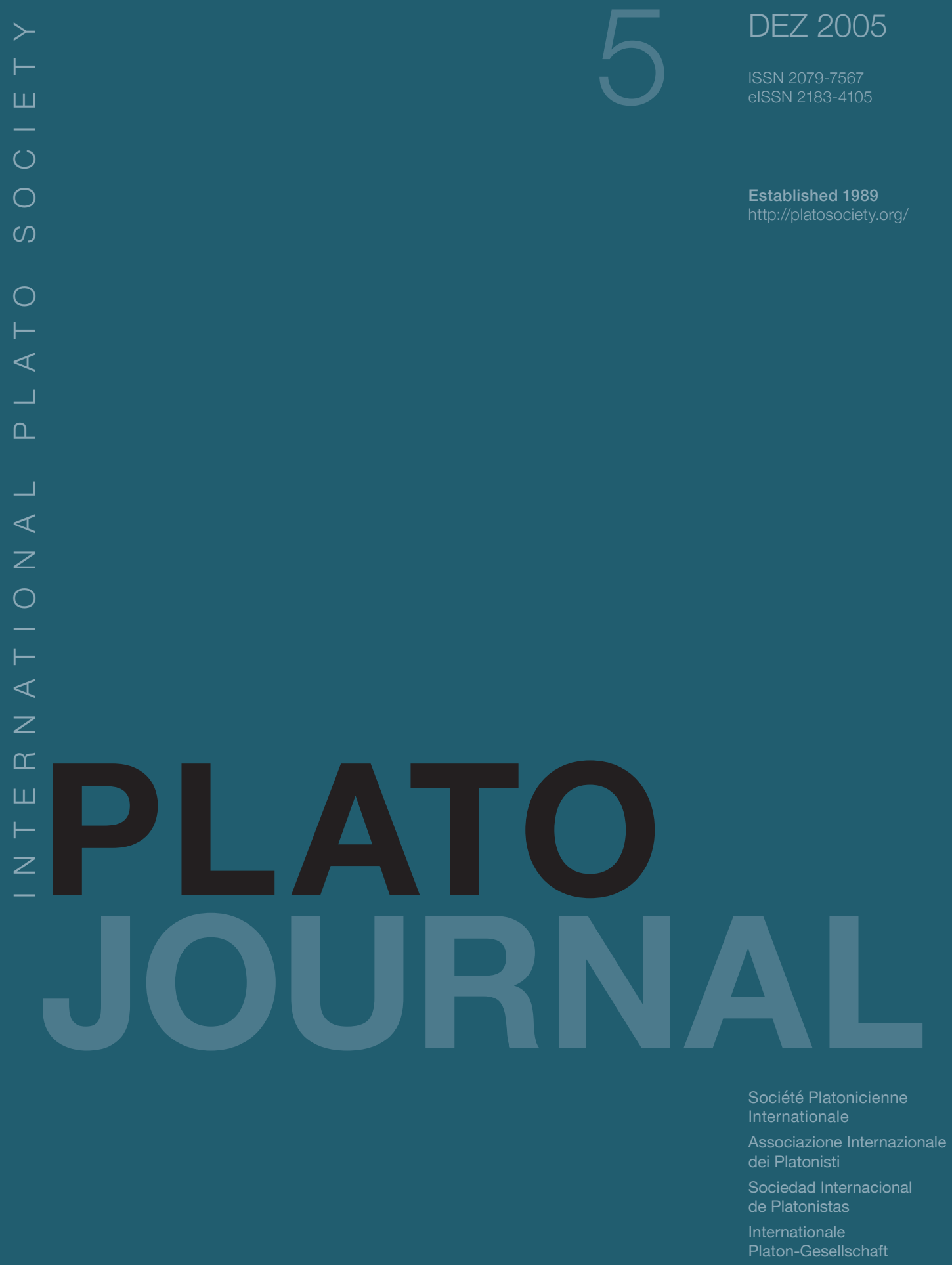




\section{LA ThÉORIE De LA Vision DANS TimÉE (45B2-D2) ET SON COMMENTAIRE PAR CALCIDIUS} $\left(\mathrm{IV}^{\mathrm{E}} \mathrm{S}\right.$. DE NOTRE ÈRE)

La vision est, chez Platon, le premier des cinq sens et occupe une place fondamentale dans sa philosophie ; d'abord en physique, car c'est l'observation de la nature qui permet d'en comprendre les secrets et mécanismes, et, ensuite, en éthique, puisque la contemplation du spectacle du monde sensible et de ses mouvements uniformes et réguliers doit conduire l'homme à adopter un mode de vie stable et bon ${ }^{1}$.

$\mathrm{Si}$, pendant longtemps, on a pu déplorer que la définition de la vision donnée dans le Timée n'ait guère retenu l'attention de la critique, cette indifférence est aujourd'hui réparée depuis la publication de la thèse d'Anne Merker sur La vision chez Platon et Aristote ${ }^{2}$. Pour autant néanmoins, la postérité platonicienne n’est guère étudiée par A. Merker, pour un texte qui a été largement transmis et glosé ; un certain nombre d'interprétations s’y sont greffées en effet, qui attribuaient à Platon, à tort ou à raison, telle ou telle théorie de la vision. Il peut être dès lors intéressant de faire le point à la fois sur le texte, ses difficultés et son sens et sur les conditions de sa réception.

\section{TIM. 45B2-D2 : Un PASSAGE DIFFICILE}

Timée traite de la vision dans le cadre de la création du corps humain par les dieux. La définition qui nous intéresse ici sera immédiatement suivie d'un développement sur le principe de réflexion des miroirs et, plus loin (67c4-d2), d’un paragraphe sur les couleurs.

En quelques phrases sont définis le vecteur de la vision et son mécanisme :

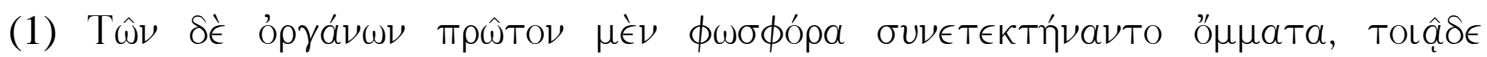

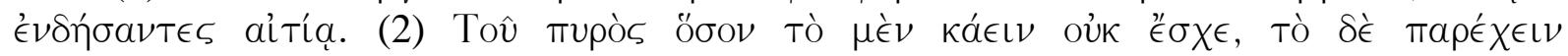

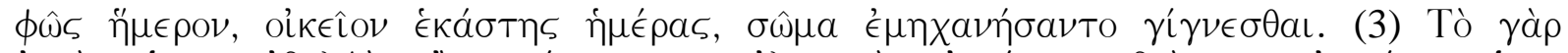

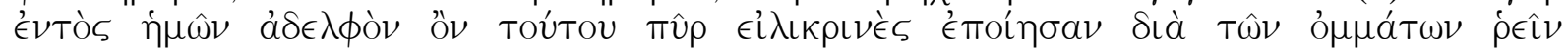

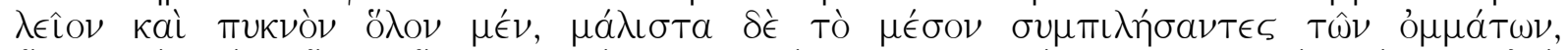

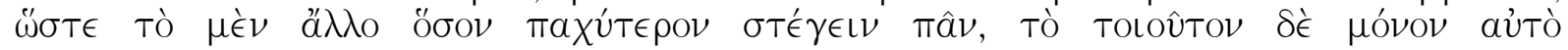

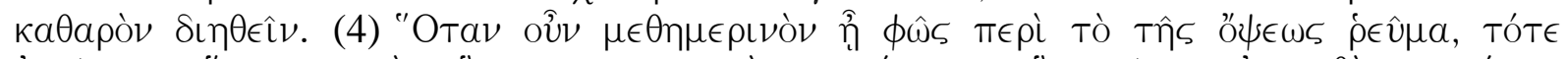

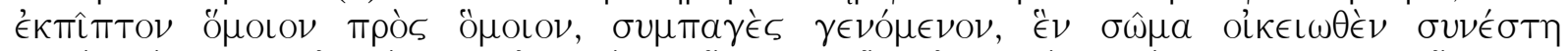

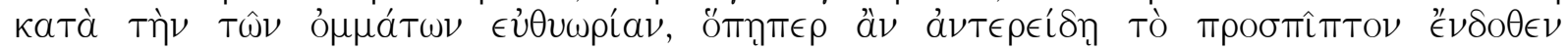

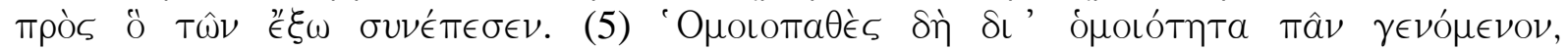

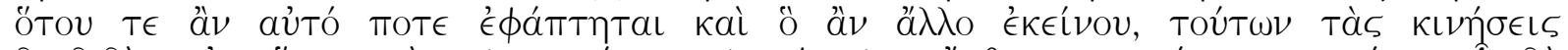

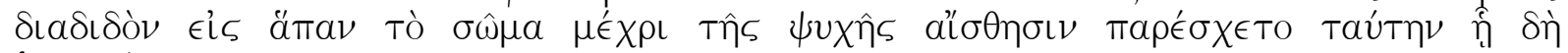
óôv $\phi a \mu \epsilon \nu$.

«(1) Entre tous ces instruments, ce sont les yeux porteurs de lumière qu'ils ont fabriqués en premier lieu ; et voilà pourquoi ils les ont fixés (dans le visage). (2) Tout ce qu'il y a de feu qui ne brûle pas, mais qui procure une douce lumière, les dieux l'ont fabriqué afin que, pour chaque jour, il devînt un corps qui leur fût apparenté. (3) En effet, le feu qui, audedans de nous, est le frère du précédent, ils firent qu'il s'écoulât à travers nos yeux dans toute sa pureté. Pour cela, procédant à la manière d'un foulon, ils ont confectionné une boule

\footnotetext{
${ }^{1}$ Cf. Tim. 47a1-c4.

${ }^{2}$ Academia Verlag, Sankt Augustin, 2003.
} 
lisse et compacte surtout en son centre, qui arrête tout autre feu plus grossier, et qui ne laisse filtrer que le feu pur, le seul de cette espèce. (4) Quand donc la lumière du jour entoure le flux issu des yeux ${ }^{3}$, alors le feu intérieur qui s'échappe, le semblable allant vers le semblable, après s'être combiné avec la lumière du jour se constitue en un seul corps ayant les mêmes propriétés tout le long de la droite issue des yeux, quel que soit l'endroit où le feu qui jaillit de l'intérieur entre en contact avec le feu qui provient des objets extérieurs. (5) Il se forme ainsi un tout qui a des propriétés uniformes en raison de son homogénéité ; si ce tout vient à entrer en contact avec lui, il en transmet les mouvements à travers tout le corps jusqu'à l'âme, et nous procure cette sensation grâce à laquelle précisément nous disons que "nous voyons" »".

D’emblée, (1) la qualification des yeux « porte-lumière » n'est pas neutre : elle explicite déjà ce qui suit. (2) Mais, si le " feu » visuel est nettement distingué de la flamme (le feu qui brûle), le sens de la phrase n'en est pas moins opaque à cause de la construction du groupe

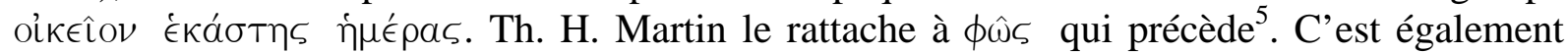
l'interprétation de A. E. Taylor qui souligne l'erreur de R. D. Archer-Hind construisant le groupe comme dépendant de $\sigma \hat{\omega} \mu \alpha^{6}$. F. M. Cornford, à partir de la même analyse, traduit: " the proper (body) of each day " ${ }^{7}$. A. Rivaud isole le génitif pour en faire un complément de temps : «... (ils ont fait) qu'elle devînt, chaque jour, un corps approprié »" . Quant à L. Brisson, tout en conservant la construction de R. D. Archer-Hind, il glose ainsi sa traduction : "Chaque jour “a” un corps qui lui est propre, étant donné que, considéré en lui-même, il constitue une totalité. C'est le feu qui, diffusé dans l'air, rend compte de la lumière du jour et qui, au coucher du soleil, se retire (cf. infra 45d) » (n. 296). On le voit, la phrase pose des problèmes d'interprétation, par le flou même de la description. En outre les échos phoniques

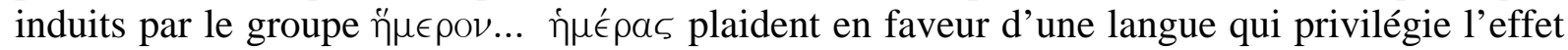
au détriment de l'exactitude scientifique. (3) Les images dans la phrase qui suit (å $\delta \in \lambda \phi o ̀ v . .$. $\sigma \cup \mu \pi \imath \lambda \eta ́ \sigma \alpha \nu T \in \zeta)$ confirment le refus platonicien de la technicité au profit d'un exposé plus expressif que précis ; quelle partie de l'œil en effet sert de filtre? L'imprécision ne fait que s'accentuer quand il s'agit du mécanisme de la vision. (4) Là encore, le seul terme un peu

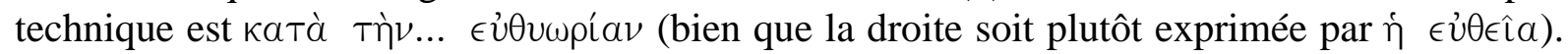
C'est cependant cette expression qui permet de voir dans le «flot » de la lumière de l'œil $(\dot{\rho} \in \hat{\imath} \nu \ldots . \hat{\rho} \in \hat{v} \mu \alpha)$ un rayon ou un faisceau plutôt qu'un halo ou un nuage. Juste avant,

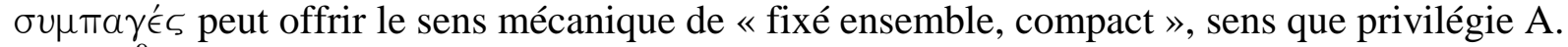
Merker ${ }^{9}$, en insistant sur le sémantisme d' "assemblage, emboîtement exact et solidité »

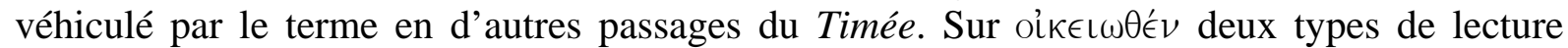
s'opposent : le terme exprime soit l'homogénéité du corps de la vision - c'est l'interprétation de A. Rivaud, F. M. Cornford et L. Brisson -, soit l'adéquation de ce corps au nôtre, comme le pense A. Merker ${ }^{10}$. Enfin, dans les dernières phrases, le choix des termes exprime de façon stylistiquement insistante à la fois l'homogénéité du composé des deux lumières - l'interne et

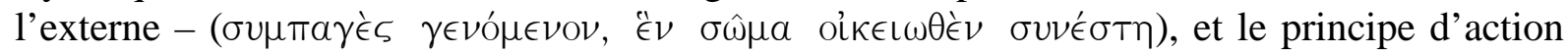

\footnotetext{
${ }^{3}$ Th. H. Martin a traduit par « le courant du feu visuel », Notes sur le Timée, note LI, p. 157-163.

${ }^{4}$ Platon, Timée / Critias, trad. L. Brisson, Paris, 1995 ², p. 140-141.

${ }^{5}$ Études sur le Timée de Platon, Paris, 1841 (réimpr. Paris, 1981), p. 123 : « (lumière) qui ne manque jamais de nous éclairer tous les jours ».

${ }^{6}$ A Commentary on Plato's Timaeus, Oxford, rééd. 1962, p. 277, avec citation de la traduction de l'éditeur anglais : « body appropriate to day-light ».

${ }^{7}$ Plato's Cosmology, p. 152 et n. 2 ; interprétation également suivie par A. Merker, p. 24 et n. 2.

8 Platon, Euvres complètes t. X, Timée - Critias, Paris, 1956, p. 162.

${ }^{9}$ La vision..., p. $24-25$ et n. 4.

${ }^{10}$ La vision..., p. 25, n. 5.
} 


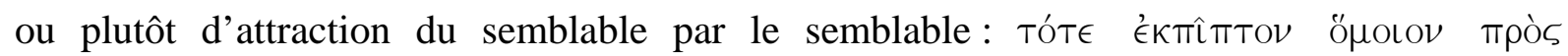

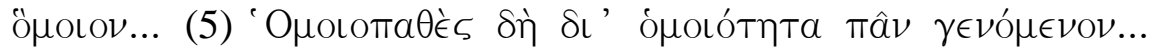

Ce passage, en dépit d’une apparente clarté générale, pose dans le détail, on le voit, de

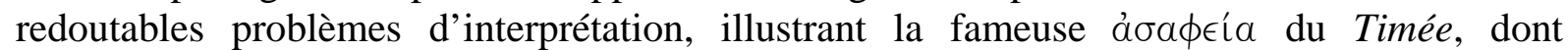
témoigne déjà, dans le monde latin, Cicéron ${ }^{11}$ et à laquelle souscrira plus tard Calcidius en l'expliquant par la technicité d'un texte que ne saurait comprendre le lecteur moyen : Timaeus Platonis et a ueteribus difficilis habitus atque existimatus est ad intellegendum, non ex imbecillitate sermonis obscuritate nata - quid enim illo uiro promptius? -, sed quia legentes artificiosae rationis, quae operatur in explicandis rerum quaestionibus, usum non habebant stili genere sic instituto, ut non alienigenis sed propriis quaestionum probationibus id quod in tractatum uenerat ostenderetur ${ }^{12}$.

Ici en l'occurrence, c'est plutôt l'absence de technicité du passage qui en rend la réception délicate. Cet extrait du Timée n’étant pas traduit par l'Arpinate, la seule version latine que nous ayons conservée est celle que Calcidius $\left(\mathrm{IV}^{\mathrm{e}}\right.$ siècle de notre ère) adresse à son ami Osius :

E quibus primi luciferi oculorum orbes coruscant, hac de causa dati. (2) Duae sunt, opinor, uirtutes ignis, altera edax et peremptoria, altera mulcebris innoxio lumine. Huic igitur, ex qua lux diem inuehens panditur, domesticum et familiare corpus oculorum diuinae potestates commentae sunt, (3) intimum siquidem nostri corporis ignem, utpote germanum ignis perlucidi sereni et defaecati liquoris, per oculos fluere ac demanare uoluerunt, ut per leues congestosque et tamquam firmiore soliditate probatos orbes luminum, quorum tamen esset angusta medietas subtilior, serenus ignis per eandem efflueret medietatem. (4) Itaque cum diurnum iubar applicat se uisus fusioni, tunc nimirum incurrentia semet inuicem duo similia in unius corporis speciem cohaerent, quo concurrunt oculorum acies emicantes quoque effluentis intimae fusionis acies contiguae imaginis occursu repercutitur. (5) Totum igitur hoc similem eandemque sortitum passionem et ob indifferentem similitudinem eiusdem passionis effectum, cum quid aliud tangit uel ipsum ab alio tangitur, tactuum motu diffundens se per omne corpus perque corpus usque ad animam porrigens sensum efficit qui uisus uocatur.

Si on compare cette traduction avec le modèle grec, un certain nombre de remarques s’imposent, dont voici les plus importantes :

(1) D’abord le calque sémantique - luci-feri pour $\phi \omega \sigma^{-} \phi$ ópa - est une des techniques de traduction plusieurs fois adoptée par Calcidius.

(2) La double qualification platonicienne (négative et positive) du feu corporel est transposée dans un parallélisme (altera... altera) qui la fausse et modifie en outre la structure

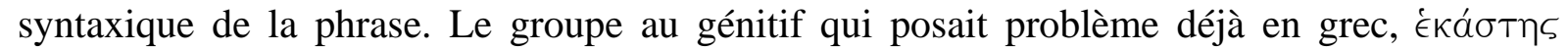

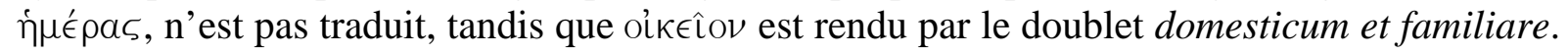
Si le sujet de $\epsilon ́ \mu \eta \chi \alpha \nu \eta ́ \sigma \alpha \nu \tau o$ (dont la traduction par commentae sunt gomme la dimension

\footnotetext{
${ }^{11}$ Cf. par exemple, De fin. II, 5, 15 : ... rerum obscuritas non uerborum facit ut non intellegatur oratio, qualis est in Timaeo Platonis.

${ }^{12}$ Comm. au Tim. c. 1 ; pour les autres références, cf. B. Bakhouche, « La transmission du Timée dans le monde latin », in Les voies de la science grecque, éd. D. Jacquart, Genève, 1997, p. 1-31 [p. 3, n. 4].
} 
mécaniste) est précisé (diuinae potestates), ce qui aide à l’intelligibilité du texte, plus ennuyeux en revanche est l'ajout malencontreux de oculorum complétant corpus $(=\sigma \hat{\omega} \mu \alpha)$, car on passe du corps de la lumière au corps de l'œil : c'est vraisemblablement la notion de corporalité appliquée à la lumière qui a induit le traducteur en erreur.

(3) Dans cette phrase, la subordination (siquidem) s'est substituée à la coordination ( $\gamma \alpha \dot{\alpha} \rho)$; des groupes sont développés (Toútov est précisé par une cascade de génitifs, et $\rho \in \hat{\imath} \nu$

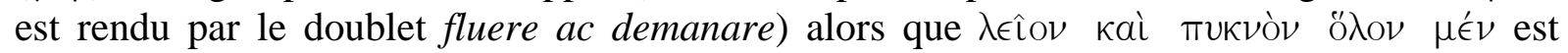
omis ; mais c'est surtout la subordination par ut qui trahit le sens du texte grec puisque l'épaississement de l'œil (cf. per leues congestosque et tamquam firmiore soliditate probatos orbes luminum) devient une qualité gratuite de cet organe de la vision et non une particularité spécifiquement vouée à une certaine fonction - servir de filtre à la lumière corporelle. La fonction téléologique de la structure de l'œil n'est donc pas conservée, pas plus d'ailleurs que sa nature qui est plus épaisse (cf. $\sigma \nu \mu \pi \iota \lambda \eta ́ \sigma \alpha \nu \tau \epsilon \zeta)$ et non «plus subtile » (subtilior) en son milieu $^{13}$.

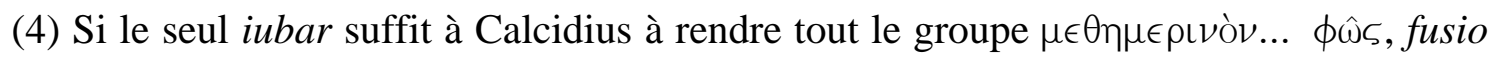
en revanche calque parfaitement le grec $\hat{\rho} \in \hat{v} \mu \alpha$. L'omission de la seule indication vaguement

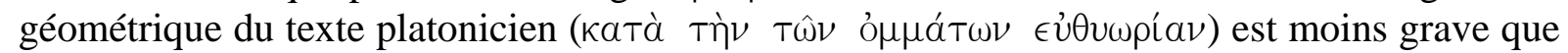
le fait de négliger, comme le fait le traducteur, la logique explicative du grec (fusion causée par l'attraction réciproque du semblable). En outre, la fin de la phrase latine est centrée, dans une structure chiasmatique qu'affectionne particulièrement le traducteur ${ }^{14}$, sur la convergence de deux rayons - le rayon visuel (pourtant déjà évoqué et uni à la lumière ambiante) et le rayon réfléchi par l'interposition de l'objet sensible : la phrase est loin d'être claire et la construction quo... quoque contribue encore à en opacifier le sens.

(5) L’emploi répété de passio (qui n’est pas sans présenter une connotation certes stoïcienne $^{15}$ mais aussi aristotélicienne) et l'utilisation du groupe similem eandemque que Calcidius réserve généralement à la désignation du Même, c'est-à-dire de l’intelligible, rendent la version latine incompréhensible. En revanche, la dernière partie de cette phrase (cum quid-uocatur) reproduit assez fidèlement le texte grec, à deux petites inexactitudes près

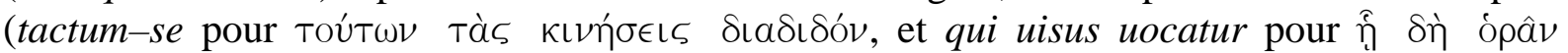
$\phi a ́ \mu \in \nu)$.

Nous avons là un bel exemple de la technique de traduction de Calcidius qui transpose plus qu'il ne traduit, tantôt contractant, tantôt dilatant le texte grec qu'il déforme en outre par l’insertion de subordination ou de compléments qui en dénaturent, au bout du compte, le sens.

\footnotetext{
13 J. Mansfeld, « Alcmaeon :'physicos' or physician ? With some remarks on Calcidius' 'On vision' compared to Galen’s Plac. Hipp. Plat. VII », in Kephalaion. Studies in Greek philosophy and its continuation offered to Professor C. J. de Vogel, J Mansfeld \& L. M. de Rijk édd., Assen, 1975, p. 26-38 [p. 29], a relevé la mauvaise construction de la phrase qu'il attribue - de façon discutable - à la source de Calcidius, et dans laquelle il

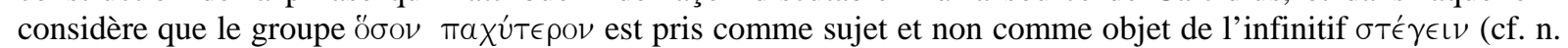
13). En réalité la structure syntaxique de la phrase grecque est complètement bouleversée, et rien n’est dit, dans la traduction latine, d'un quelconque arrêt ou blocage du feu « épais ».

${ }^{14}$ Sur le chiasme dans les traductions, cf. R. Poncelet, Cicéron traducteur de Platon. L'expression de la pensée complexe en latin classique, Paris, 1957, p. 299-300.

${ }^{15}$ Cf. J.-P. Dumont, « Sensation et perception dans la philosophie d’époque hellénistique et impériale », ANRW II. 36.4, p. 4718-4764, à propos de la théorie stoïcienne de la vision, p. 4750 : « Pour qu'il y ait sensation, il faut que l’organe sentant pâtisse du fait du sensible propre, qualité appartenant à l'objet sensible ».
} 
L'étude de la version calcidienne de ce court passage du Timée suffit donc à infirmer les conclusions, élogieuses pour l'ami d’Osius, des études de Poncelet, études déjà anciennes mais qui n'ont à ce jour jamais été véritablement remises en question, et où on peut lire par exemple :

«Le texte de Chalcidius est écrit dans une langue philosophique, parce que la phrase, quoique correcte, ignore la vanité littéraire et rencontre une beauté d'ordre intellectuel dans la coïncidence de l'expression et de l'idée ${ }^{16}$.

De même doivent être reconsidérées les analyses (encore plus anciennes) de Fr. Blatt pour qui l'ère chrétienne dans laquelle s'inscrit Calcidius ouvre la voie royale de la traduction : "Le littéralisme », écrit-il ${ }^{17}$, « est étroitement lié à la nouvelle religion » et qui voit dans la version calcidienne « la première traduction chrétienne du Timée » (p. 223), avant de conclure qu'à la différence de la traduction de Cicéron, «le texte de Chalcidius suit presque mot à mot le grec » (p. 226).

Nous avons cru pouvoir montrer que de tels éloges sont inappropriés, à tout le moins pour le passage qui nous occupe. Le lecteur de cette version latine attribuerait à Platon une théorie de la vision qui n'est absolument pas la sienne. Le texte grec est pourtant assez clair : la partie épaisse de l'œil laisse filtrer le feu intérieur le plus pur qui s'unit à la lumière environnante pour former un tout homogène ; quand ce faisceau entre en contact avec la lumière émise par un objet sensible, il en renvoie les mouvements à l'âme ; d'où la sensation. Pour Calcidius en revanche, l'œil est apparenté (!) à la lumière, le feu intérieur s'écoule par la partie subtile et non épaisse du centre oculaire (qui ne remplit dès lors plus sa fonction de sas), et, dans le faisceau lumineux et visuel, convergent le feu intérieur (n’y est-il pas déjà?) et la lumière (?) venue de l'objet sensible ; leur rencontre permet de renvoyer la sensation à l’âme.

Les déviations de la version par rapport au texte-source posent dès lors un certain nombre de problèmes : peuvent-elles s'expliquer par l'orientation du commentaire? ou, à l'inverse, induire une lecture exégétique?

\section{La ThÉorie Platonicienne De La Vision Et Son Commentaire Par Calcidius}

Les difficultés textuelles du texte platonicien se doublent de fait d'une opacité théorique, ou à tout le moins d'un manque de cohérence interne, dans la mesure où, comme cela arrive souvent avec Platon et en dépit de l'importance que Timée attribue à la vision ${ }^{18}$, le mécanisme exprimé dans le passage étudié, s’il reproduit la théorie de la République, s’écarte de celle du Théétète. Avant le Timée en effet, Platon a déjà évoqué le mécanisme de la vision : sans s'arrêter au Ménon qui présente très rapidement un résumé ironique de la théorie d’Empédocle ${ }^{19}$, il a été dit, dans le Thééthète ${ }^{20}$, que le principe de la vision vient de la

\footnotetext{
16 « Deux aspects du style philosophique latin : Cicéron et Chalcidius, traducteurs du "Phèdre” 245C », REL 28, 1950, p. 145-167 [p. 166] ; cf. également du même auteur Cicéron traducteur de Platon..., et sa thèse complémentaire, Cicéron et Chalcidius. Deux époques du style philosophique latin, Paris, 1953.

${ }^{17}$ " Remarques sur l'histoire des traductions latines », C\&M 1, 1938, p. 217-242 [p. 221].

${ }^{18}$ Cf. également 46A2-C6 sur les miroirs et 67C4-68D8 sur les couleurs. Calcidius, de même, consacrera trois sections de son commentaire à la vue : X. De uisu. XI. De imaginibus. XII. Laus uidendi (cf. c. 7), soit 32 chapitres (des chapitres 236 à 268).

${ }^{19}$ Cf. 76d5-6 et note 2 p. 243, éd. A. Croiset, Paris, 1972.
} 
rencontre de deux vecteurs lumineux, celui de l'œil et celui de l'objet vu, mais dans la République $^{21}$, il est clair que la vision vient de la convergence de ces deux rayons associée au medium de la lumière du jour. Nous retrouvons ce dernier principe dans le Timée : la vision repose sur trois types de lumière (cf. les occurrences de $\phi \hat{\omega} s$ et de ses composés dans le passage étudié), et de fait le rayon lumineux intérieur, dès qu'il sort de l'œil, constitue avec la lumière du jour un seul faisceau qui va à la rencontre de la lumière émanant des objets vus. Quand le faisceau complexe rencontre la lumière d'un objet, il se produit un phénomène de réflexion (y a-t-il d'abord fusion des trois rayons?) qui ramène le même faisceau (désormais triple), à travers l'œil, jusqu'à l'âme. On peut donc penser, bien que ce ne soit pas précisé explicitement, que, si la sensation revient à l'âme, c'est que la vision est un mouvement qui prend déjà son origine en elle.

D'un autre côté, le refus de toute explication technique permet au philosophe de poser des principes généraux, voire des axiomes, sans se préoccuper de rendre compte des processus technique et physiologique, et les explications de Timée laissent un certain nombre de questions sans réponse : où se rencontrent le rayon visuel et la lumière issue de l'objet ? à la surface de celui-ci ou à mi-distance, comme le pense A. Rivaud ${ }^{22}$ ? Par quel mécanisme se produit une réflexion de $180^{\circ}$, pour que le rayon revienne vers l'œil ? D’autre part, en dépit de la référence implicite au système d’Empédocle, le mécanisme de la vision exposé par Timée ne saurait être rattaché au système du philosophe sicilien, contrairement à ce que pense $\mathrm{A}$. $\mathrm{E}$. Taylor $^{23}$, car Empédocle supposait, au témoignage de Théophraste ${ }^{24}$, que les sensations

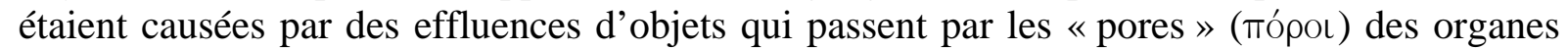
des sens : du fait de l'attraction des semblables, les effluences des objets blancs, par exemple, stimulent les conduits ignés de l'œil et ceux des objets noirs ses conduits aqueux. D'ailleurs, la physiologie de l'œil, essentielle dans le mécanisme de la vision, n'est nullement prise en compte dans le Timée. À la prendre avec sérieux ${ }^{25}$, la théorie de Platon paraît offrir un compromis entre deux systèmes concurrents : celui où la vision vient de la rencontre du rayon visuel avec l'objet vu et celui où elle résulte de l'action de l'objet vu sur l'organe de la vision $^{26}$. Nous y reviendrons.

L’important pour Platon, en réalité, est ailleurs : il est dans la place des yeux (et des autres organes des sens) au sommet du corps, dans la tête. Dans le développement précédemment cité, la phrase liminaire (1) n’a pas été assez prise en compte dans la tradition exégétique qui a suivi : ce n'est pas en effet une simple introduction; elle implique au contraire une corrélation étroite entre la façon dont le corps humain est constitué et la finalité de chacune de ses parties. Même si cela n'est pas exprimé clairement, le rôle fondamental, dans l'acte de voir, n'est pas joué par l'œil mais par l'âme, qui joue un rôle actif en décryptant ce qui est vu. On peut en déduire que celle-ci ne peut se situer que dans la tête, car l'exposé

\footnotetext{
${ }^{20}$ Théétète 156c6-c7 et cf. également 184-186.

${ }^{21}$ Rép. 507c-508a.

${ }^{22}$ Éd. du Timée, Paris, 1956, notice p. 105.

${ }^{23}$ A Commentary on Plato's Timaeus, p. 280.

${ }^{24}$ De sensu $\S 7$ = Doxographi Graeci, éd. H. Diels, Berlin, 1965, p. 500, 19-29 ; cf. D. O’Brien, « The Relation of Anaxagoras and Empedocles », JHS 88, 1968, p. 93-113, part. « V. Theories of Vision » p. 109-113.

${ }^{25}$ A. Philonenko pense en effet que Platon, « percevant la superficialité de ses explications, ... s’est amusé, et c’est un vrai problème de savoir quand et comment commence la plaisanterie » (Lectures platoniciennes, Paris, 1997, p. 533).

${ }^{26}$ Cf. D. O’Brien, « Plato and Empedocles on evil », in Traditions of Platonism. Essays in Honour of John Dillon, J. J. Cleary éd., Aldershot, 1999, p. 3-27 [p. 10].
} 
sur la vue, dans le Timée, ne peut se comprendre qu'en relation avec la place de l'âme rationnelle dans la tête, et il en est de même pour les autres organes sensoriels. Mais, faute d'un exposé liminaire sur une théorie générale de la sensation, Platon lui-même condamnait ses successeurs à considérer ses différents exposés sur les sens comme des touts. On comprend dès lors que, dans la tradition exégétique postérieure à Platon, les deux axes psychologique et physiologique - ou plutôt optique - aient été nettement séparés : d'un côté en effet, on discute de la place de l'hêgemonikon et de l'autre du mécanisme de la vision. Aristote, déjà, critique la théorie de Timée, qu'il juge - à tort, on l'a vu - identique à celle d'Empédocle et estime que ce qui prédomine dans l'œil ce n'est pas le feu, ni la lumière, mais l'eau $^{27}$ : il inaugure ainsi une longue suite de remarques axées le plus souvent sur le seul vecteur de la vision. Le rapport avec l'âme est rarement fait, et la dimension téléologique du système platonicien est mal perçue par les exégètes ou les doxographes qui ont suivi.

De fait, la finalité gnoséologique de la vision n'apparaît guère dans le commentaire de Calcidius qui consacre les chapitres 236-248 (associés par les éditeurs à la section De uisu) à l'exégèse de Tim. 45B2-D2. Dans un premier temps, il énumère les principales théories de la vision et de la perception des couleurs ou des objets, c'est-à-dire qu'il se réfère à l'origine et au résultat de l'acte de voir mais non à son mécanisme.

Pour les atomistes, d'abord définis par une périphrase résumant leur théorie, on voit grâce aux émanations lumineuses venant des objets et qui arrivent au siège des sens :

Omnes qui rerum initia corpora censuerunt uel coetu innumerabilium minutorum congesto inani uel perpetuorum continuata proceritate, dicunt uidere nos simulacrorum incursionibus; fluidam quippe materiem formatas iuxta sui similitudinem exudare subtiles corporum fusiones, quae sunt uisibilium simulacra rerum, eaque cum uisus noster incurrerit, hausta et recepta meatibus transmittat ad eum per quem sentimus spiritum (c. 236).

Si certaines expressions ne peuvent se comprendre que comme des traductions du grec,

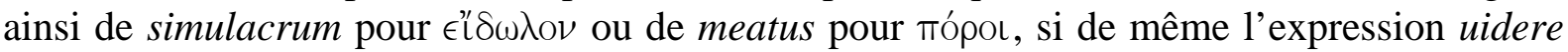
nos simulacrorum incursionibus n'est pas sans rappeler Diogène Laërce à propos des idées de

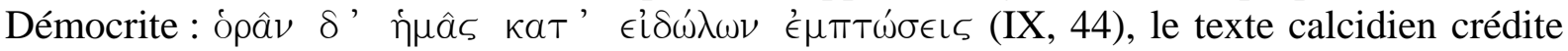
les atomistes d'une théorie qui donne à l'intelligence un rôle plus actif que ce dont les créditent les doxographes :

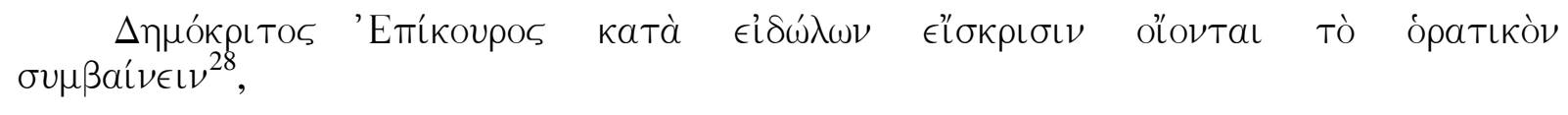

mais qui est conforme à ce qu’on peut lire dans la Lettre à Hérodote ${ }^{29}$.

Vient ensuite la rapide évocation - en des termes à coloration stoïcienne - du système d'Héraclite ${ }^{30}$ dans lequel le rayon parti de l'œil touche l'objet :

\footnotetext{
${ }^{27}$ De sensu, c. 2, 437b11sqq. Sur la théorie de la vision chez Aristote, cf. A. Merker, La vision..., p. 133-242.

${ }^{28}$ Doxogr. Graeci, éd. H. Diels, Berlin, 1965², p. 403 : Plutarque Epit. IV, 13 = Sobée Ecl. I, 52 [44].

${ }^{29}$ Lettre à Hérodote $\S 49$ = Diogène Laërce, Vies, X, 49.

${ }^{30}$ Sur Héraclite, voir Diogène Laërce, Vies IX, 1-17, mais on n’y trouve rien sur sa théorie de la vision. J. H. Waszink (note p. 249) rapproche - sans raison selon nous - le passage calcidien d'une phrase tirée de Sextus

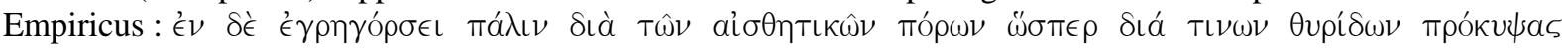


At uero Heraclitus intimum motum, qui est intentio animi siue animaduersio, porrigi dicit per oculorum meatus atque ita tangere tractareque uisenda.

Cette phrase devrait figurer dans les Fragmente der Vorsokratiker de Hermann Diels, puisqu'elle est le seul témoignage que nous ayons sur la théorie de la vision du philosophe d'Éphèse. En revanche, la définition stoïcienne qui suit trouve, elle, sa place parmi les Stoicorum veterum fragmenta de J. von Arnim :

Stoici uero uidendi causam in natiui spiritus intentione constituunt, cuius effigiem coni similem uolunt. Hoc quippe progresso ex oculorum penetrali, quae appellatur pupula, et ab exordio tenui, quo magis porrigitur, in soliditatem opimato exordio, penes id quod uidetur locata fundi omnifariam dilatarique uisus illustratione ${ }^{31}$.

Vient ensuite tout un exposé sur le rapport entre la distance du cône à l'œil et la netteté de la vision, illustré par une série d'exemples d’illusions d’optique. Dans le passage précédemment cité, l'accent est mis sur l'origine oculaire de la vision, mais le principe même du résumé doxographique trahit quelque peu la théorie du Portique : on reconnaît mal, dans le natiuus spiritus (qui traduirait un $\sigma u ́ \mu \phi v т о \nu ~ \pi \nu \in \hat{v} \mu \alpha$ ) le $\pi \nu \in \hat{v} \mu \alpha$ stoïcien comme vecteur indispensable de la vision, encore moins le ảń $\rho$ de la définition d'Alexandre d'Aphrodisie qui

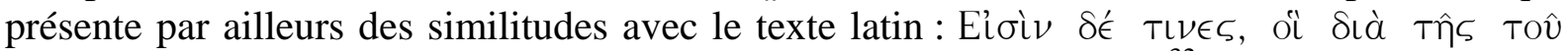

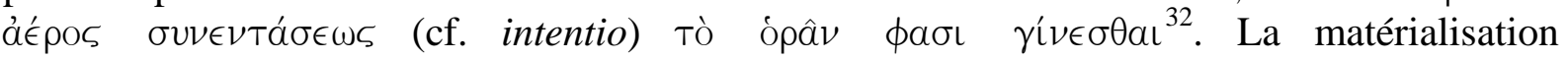
géométrique de ce souffle comme sommet d'un cône induit en outre l'image d'un faisceau enveloppant l'objet de la vision.

Enfin les « géomètres et les péripatéticiens » se voient attribuer une théorie commune :

Geometrae cum Peripateticis concinentes radii effusione uisum operari putant, cum per fulgidam lucidamque pupulae stolam in directum emicans radius serenam porrigit lineam, quae gyris oculorum circumuecta motibus dispergat undique lucem contemplationis... Ergo notae geometricae comparant quod uidetur et illuc uersum ferri censent uisus intentionem... (c. 238).

La vision part donc de l'œil sous la forme géométrique d'un vecteur (radius) dont on ignore la nature (est-il lumineux ?) ; ce vecteur est orienté vers l’objet qui, lui, est présenté comme un point (nota). Si cette figuration géométrique permet d'associer les géomètres aux philosophes, la paternité de cette théorie est une fois de plus problématique et sa source péripatéticienne est particulièrement sujette à caution. Nemesius évoque lui aussi les " géomètres ", mais c'est un système de faisceau conique qu'il leur attribue avec une certaine brièveté $^{33}$. Dans le commentaire calcidien, la théorie calque plutôt le système stoïcien et, s'il fallait distinguer les deux, il conviendrait d'associer la présente définition de l'exégète latin à un fuseau (qui se dilate puis se contracte sur l’objet de la vision associé au point). Par ailleurs, contemplationis paraît traduire un terme comme ő\&ıs en trahissant la polysémie du grec, et le

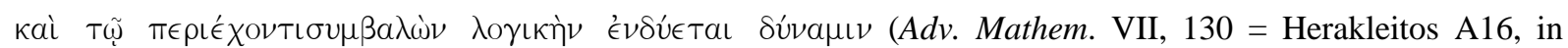
Fragm. der Vors., H. Diels, p. 148).

${ }^{31}$ C. $237=$ StVF II, 863.

${ }^{32}$ St.V.F. II 864 = Alexander Aphrod. de anima libri mant. p. 130, 14 Bruns.

${ }^{33}$ Nemesii Emeseni De natura hominis, éd. M. Morani, Leipzig, 1987, c. 7. 
seul parallèle qu'on puisse trouver dans la tradition doxographique grecque est attribué à

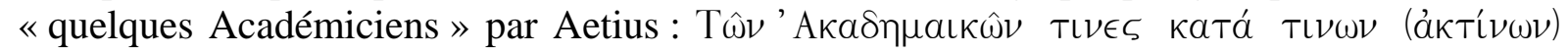

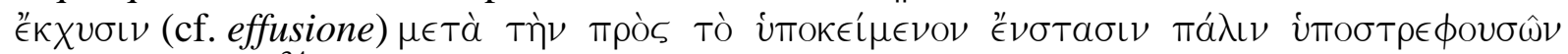

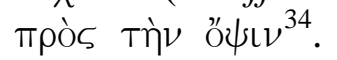

Après cinq chapitres consacrés à différentes sortes de vision et d'altération de la vision, Calcidius revient, dans les quatre derniers chapitres de la section, au texte même de Platon. Dans un chapitre liminaire, le commentateur mêle des paraphrases du Timée - de la page 45 mais aussi, de façon anticipée, de la page 62 -, et du passage de la République où l'œil est comparé au Soleil. Néanmoins, les trois rayons nécessaires pour voir sont bien présents dans le résumé du chapitre 245 :

Tribus ergo his concurrentibus uisus existit trinaque est ratio uidendi: lumen caloris intimi per oculos means, quae principalis est causa, lumen extra positum, consanguineum lumini nostro, quod simul operatur et adiuuat, lumen quoque quod ex corporibus uisibilium specierum fluit, flamma seu color, qui perinde est atque sunt omnia quibus propositum opus effici non potest, ut sine ferramentis quae sunt operi faciendo necessaria; quorum si quid deerit, impediri uisum necesse est.

Ce passage est sans ambiguïté : il définit avec exactitude le principe de la vision selon les platoniciens, puisqu'il s'agit de trois "lumières" (lumen) qui se mêlent, et le calor intimus qui est émis par le corps confirme le sens de «feu visuel » que Th. H. Martin donnait déjà à ő $\psi \iota \varsigma^{35}$. La sensation se présente donc comme un mouvement (cf. concurrentibus), conformément à la définition que donne L. Brisson de la sensation d'après le Timée : "La sensation est affaire de communication. Ce qui est communiqué c'est la propriété que manifeste un objet, par l'intermédiaire d'un mouvement qui trouve sa source à l'extérieur. Et la transmission de ce mouvement se fait de façon mécanique de partie à partie, par une circulation à travers le vivant en son entier, corps et âme ${ }^{36}$. Mais le mécanisme de la vision - le rapport et le parcours des trois «lumières » - n'est pas explicité. Cette absence de précision peut s'expliquer par la fonction de transition que remplit ce rappel de l'orthodoxie platonicienne, car la référence au dernier groupe, dans le chapitre qui suit, s'inscrit dans une perspective polémique, à travers une attaque étrangement violente contre les iuniores philosophi :

... Iuniores philosophi, ut non optimi heredes paternum censum in frusta dissipantes perfectam atque uberem sententiam, in mutilas opiniunculas ceciderunt. Quare faciendum ut ad certam explorationem Platonici dogmatis commentum uetus aduocetur medicorum et item physicorum, illustrium sane uirorum, qui ad comprehendendam sanae naturae sollertiam artus humani corporis facta membrorum exectione rimati sunt, quod existimabant ita demum se suspicionibus atque opinionibus certiores futuros, si tam rationi uisus quam uisui ratio concineret. Demonstranda igitur oculi natura est de qua cum plerique alii tum Alcmaeo Crotoniensis, in physicis exercitatus quique primus exectionem aggredi est ausus, et Callisthenes, Aristotelis auditor, et Herophilus multa et praeclara in lucem protulerunt : duas esse angustas semitas quae a cerebri sede, in qua est sita potestas animae summa et

\footnotetext{
${ }^{34}$ Dox. Graeci, p. 403.

${ }^{35}$ Cf. supra n. $2 *$.

${ }^{36}$ "Perception sensible et raison dans le Timée », in Interpreting the Timaeus - Critias, T. Calvo \& L. Brisson édd., Sankt Augustin, 1997, p. 307-316 [p. 311].
} 
principalis, ad oculorum cauernas meent naturalem spiritum continentes; quae cum ex uno initio eademque radice progressae aliquantisper coniunctae sint in frontis intimis, separatae biuii specie perueniant ad oculorum concauas sedes, qua superciliorum obliqui tramites porriguntur, sinuataeque illic, tunicarum gremio naturalem humorem recipiente, globos complent munitos tegmine palpebrarum, ex quo appellantur orbes... (c. 246).

Qui sont ces philosophes si violemment pris à parti ? Pourquoi le sont-ils ? Quelles idées professaient-ils ? Cela n’est pas précisé, mais le recours aux autorités médicales qui ont pratiqué des dissections permet au commentateur d'exposer l'anatomie de l'œil relié au cerveau pour confirmer "leurs hypothèses et conjectures, si la vue s'accordait autant à la raison que la raison à la vue ". S' « il y a deux passages étroits qui, du siège du cerveau où est placée la puissance dirigeante de l'âme (potestas animae summa et principalis = ì $\gamma \in \mu$ ovıкóv), arrivent aux globes oculaires en transportant le souffle naturel (naturalem spiritum = бú $\mu \phi \cup т о \nu \pi \nu \in \hat{v} \mu \alpha)$ ), cela prouve, par delà une structure anatomique de l'œil plutôt primitive, que, pour que l'objet vu puisse être identifié, il faut le concours de l'intelligence, c'est-à-dire de la partie de l'âme placée dans le cerveau. C'est donc que le lieu de l'âme raisonnable est le cerveau. Si la logique du raisonnement n'est pas apparente, le rapport entre la structure de l'œil, le mécanisme de la vision et le rôle du cerveau est en revanche formulé avec netteté.

Dès lors, il est vraisemblable que les penseurs ainsi attaqués par Calcidius refusaient le lien entre la vue et le cerveau, refusaient le principe d'une transmission visuelle au cerveau, quelle que soit la modélisation de nature physico-géométrique adoptée. Ils rejetaient donc toutes les théories précédemment évoquées qui offraient une approche tactile de la vision. Or Aristote déjà faisait de ce sens, comme de toute sensation, un processus passif, mais il ne saurait entrer, chronologiquement, dans la catégorie des iuniores et, d'autre part, les péripatéticiens ont déjà été nommés. En revanche, les sceptiques, s’opposant aux dogmatiques, considéraient que la nature des choses ne peut être appréhendée, par quelque organe que ce soit, de façon absolue et que les sens sont trompeurs. "Le pyrrhonisme » en effet, comme le souligne J.-P. Dumont ${ }^{37}$, « repose sur une gnoséologie sceptique qui substitue à l'intellect d'une part et aux sens d'autre part le critère du phénomène ». De même, les néoplatoniciens ont adopté, pour la vision en particulier, une attitude différente de celle de l’École, en récusant la nécessité d’un milieu pour la vision et en faisant paradoxalement de l'objet le sujet de la vision. Plotin écrit en effet :

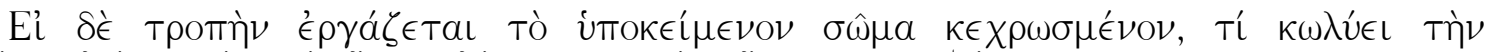

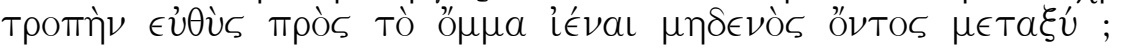

« Mais si c'est l'objet, le corps coloré, qui agit en produisant une modification, qui empêche que cette modification arrive instantanément à l'œil, sans milieu intermédiaire... ? »,

rendant ainsi l'œil totalement passif et gommant le rapport de l'œil au cerveau. La vision s'expliquerait donc par l'influence sympathique de la lumière extérieure sur celle de l'œil, sans nécessité aucune d'un medium. Qui est donc visé par Calcidius ? Il est trop tôt pour répondre à cette question.

\footnotetext{
37 « Sensation et perception dans la philosophie... », p. 4753.
} 
Enfin, dans les deux derniers chapitres de la section (c. 247-248) ${ }^{38}$, l'exégète revient au texte du Timée, en un commentaire paraphrastique ${ }^{39}$ qui nous semble de fait influencé par une autre source que le développement doxographique précédent. Sans que les erreurs de traduction relevées plus haut aient une incidence sur l'interprétation globale du passage, Calcidius résume la position orthodoxe de l'école, en explicitant la théorie platonicienne de la vision par la coalescence de deux rayons lumineux, celui de l'homme et celui du jour, pour constituer un vecteur homogène qui se réfléchit à la surface de l'objet vu : l'exégète va au delà du texte platonicien, en précisant ici le lieu de la rencontre des rayons.

Si l'on voit bien la méthode utilisée ici - comme dans bien d'autres sections du commentaire - par l'exégète latin et qui consiste dans des exposés doxographiques accompagnés de " commentaires » au texte platonicien, la question qui se pose maintenant est celle de la part de l'auteur dans son commentaire et de sa place dans la chaîne exégétique.

\section{L’INSCRIPTION DE CALCIDIUS DANS LA CHAÎNE EXÉGÉTIQUE}

Calcidius occupe assurément une place originale dans la tradition platonicienne tardive, par une double approche du Timée : une approche doxographique, très générale mais, on l'a vu, assez fidèle, et une approche exégétique de type paraphrastique.

\section{L'approche exégétique}

J. H. Waszink considère que l'ensemble des chapitres étudiés offre un écho du développement de Porphyre dans son traité Sur les sens, et dont il aurait donné une forme abrégée dans son commentaire au Timée ${ }^{40}$. De même A. R. Sodano rattache au Commentaire au Timée de l'élève de Plotin les chapitres 244-248 qui en constituent le fragment $87^{41}$.

Pourtant la typographie adoptée par l'éditeur du texte calcidien pour les citations du Timée montre bien, dès le premier regard, le départ entre commentaire et doxographie et effectivement un changement de technique au c. 247, où le commentateur revient pour quelque temps à l'analyse du texte grec, dans sa version latine naturellement. Or la paraphrase calcidienne reste fondamentalement fidèle à l'orthodoxie platonicienne et s'écarte de facto de la théorie porphyrienne qui fait de la perception une auto-perception par l'âme, du fait de l'identité qui existe entre elle et les objets qu'elle voit, comme en témoigne Nemesius au c. 7 de son De natura hominis :

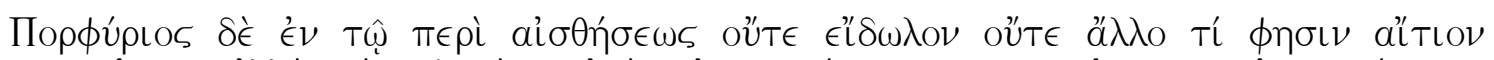

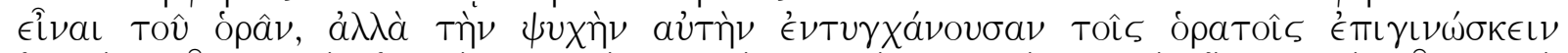

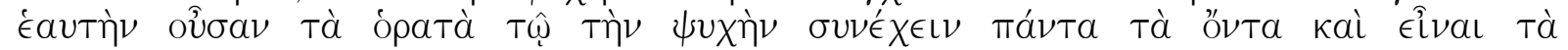

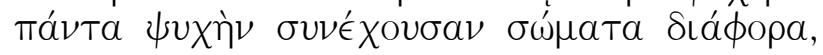

\footnotetext{
${ }^{38}$ Mais également dans les deux premiers de la section suivante « Sur les images », ce qui signale le caractère artificiel des découpages établis par les éditeurs J.H. Waszink comme, avant lui, J. Wrobel.

${ }^{39}$ Sur la paraphrase comme commentaire voir H. von Staden, « “A woman does not become ambidextrous ?” : Galen and the culture of Scientific Commentary », in The Classical Commentary. History, Practices, Theory, Roy K. Gibson \& Christina Shuttleworth Kraus édd., Brill, Leyde-Boston-Cologned, 2002, p. 109-139.

${ }^{40}$ Praefatio p. xcii-xciii.

${ }^{41}$ Porphyrii In Platonis Timaeum commentariorum fragmenta, Naples, 1964, p. 104-107.
} 
«Porphyre affirme, dans son traité De la Sensation, que ce n’est ni le cône ni le simulacre ni toute autre chose qui est la cause de l'acte de voir, mais c'est l'âme elle-même qui, en rencontrant les objets visibles, reconnaît qu'elle est elle-même ces objets visibles, parce que l'âme contient en elle-même tous les étants ${ }^{42}$.

En supprimant tout intermédiaire optique entre l'âme et l'objet, Porphyre va donc plus loin que les stoïciens pour qui la cause principale de la vision était déjà l'hêgemonikon. Le témoignage de Nemesius souligne fermement l'intérêt du philosophe néoplatonicien pour le mécanisme psychologique de la vision plus que, comme nous le trouvons chez Calcidius, pour la physiologie de la sensation.

\section{L'approche doxographique}

Quant à la partie doxographique, il faut en relever d'emblée l'originalité qui s'exprime d'abord par le souci d'exhaustivité clairement affiché par le commentateur au chapitre 236 :

Sed quoniam de hoc plerique alii post ipsum opiniones uarias libris conditis sunt executi, eas quae sunt in honore perstringam, quo perfectior propositae rei tractatus habeatur.

De fait, la doxographie calcidienne est de loin la plus développée (dix pages dans l'édition Waszink) que nous puissions trouver dans ce genre de littérature, aussi bien dans le monde grec que latin.

Assurément, nous retrouvons des parallélismes ponctuels avec Aetius ou Nemesius, qui illustrent un même fonds doxographique. Assurément, la source de Calcidius est grecque, à voir les calques linguistiques déjà relevés, mais le brouillage lexical - aussi bien pour les différentes définitions de la vision que pour qualifier la partie supérieure de l'âme (cf. c. 246 : potestas animae summa et principalis) - paraît signaler une influence stoïcienne. Il convient toutefois de minorer la coloration, signalée plus haut, stoïcisante de la théorie d'Héraclite car ce philosophe a été tenu en grande considération par les philosophes du Portique et, un peu plus loin ${ }^{43}$, Calcidius associe encore le philosophe d'Éphèse aux stoïciens. Il n'est donc pas impossible qu’Héraclite ait déjà été lié aux stoïciens dans la source de l'exégète.

La piste porphyrienne suggérée par J. H. Waszink et A. R. Sodano n'est pas davantage abandonnée par J. Mansfeld qui annonce d'emblée, au vu de nombreux parallèles entre la section Sur la vision de Calcidius et le livre VII du De Placitis Hippocratis et Platonis de Galien, une source commune. Et d'ajouter : «The author of the exposition used by Galen and Calcidius (or rather, Calcidius' immediate source, i.e., probably, Porphyry) argued that Plato's theory that the rational soul... is the centre of sense-perception, is correct... ${ }^{44}$.

\footnotetext{
${ }^{42}$ Traduction de P. Hadot dont nous reproduisons également le soulignement par les italiques pour la phrase centrale de cet extrait, Porphyre et Victorinus I, Paris, 1968, p. 238.

${ }^{43}$ C. 251 : Heraclitus uero, consentientibus Stoicis, rationem nostram cum diuina ratione conectit regente ac moderante mundana...

44 «Alcmaeon :'physicos’ or physician? ... », p. 26. Voir de même les citations de Calcidius parmi les testimonia in C. J. Larrain, Galens Kommentar zu Platonis Timaios, Teubner, Stuttgart, 1992 : fr. 27 // c. 237 (p. 161-162), fr. 22 // c. 245 (p. 142-143), et fr. 18 // c. 247 (p. 134).
} 
Il est vrai que le commentateur latin témoigne d'un intérêt particulier pour le domaine médical $^{45}$, et sa complaisance à décrire l'anatomie de l'œil plaiderait plus en faveur d'une source philosophico-médicale. Si W. W. Jaeger, comparant Nemesius-Porphyre, CalcidiusPorphyre et Galien, en a conclu à une source doxographique commune du II $^{\mathrm{e}}$ siècle de notre ère ${ }^{46}$, cette source pourrait, sinon se confondre avec le médecin de Pergame, du moins allier comme lui intérêt marqué à la fois pour la philosophie et pour la médecine. D’ailleurs la réfutation acerbe des iuniores philosophi par référence à la dissection de l'œil pourrait être lue comme un écho d'une violente polémique tout autant philosophique que médicale.

\section{L’originalité de Calcidius}

L'aspect polémique du paragraphe consacré aux iuniores philosophi donne à Calcidius une place à part dans la tradition doxographique, généralement neutre et didactique. Ce dernier trait nous permet-il de voir dans l'exégète un témoin de son temps ? ou du moins d'une époque?

Il est le seul en effet à évoquer un certain nombre de théories comme celle d'Héraclite ou comme, aux chapitres 239-242, les différents types de vision - phasis, emphasis et paraphasis qui est déjà un hapax en grec -, voire le développement sur la dissection de l'œil attribuée, entre autres, à Alcméon de Crotone. Galien, lui aussi, se réfère à la dissection pour prouver le lien entre la vue et le cerveau, mais, s’il cite volontiers Érasistrate, il ne nomme aucune autre autorité médicale ${ }^{47}$, et Alcméon, quand il est cité par les doxographes, c'est de façon très brève comme chez Stobée :

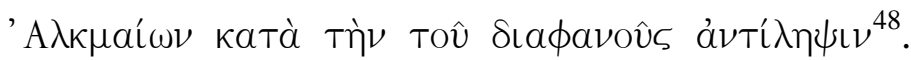

Par ailleurs, l'acrimonie du commentateur quand il s'en prend aux iuniores philosophi pourrait se lire - répétons-le - comme l'écho d’une querelle entre écoles médicales. L'exégète latin adopterait ici une attitude anti-méthodique dans la mesure où la base philosophique de cette école médicale est le scepticisme ${ }^{49}$. Le phénoménisme sceptique dont on a déjà parlé a pour corollaire, en médecine, l’indifférence au caché. D’où sans doute la nécessité de révéler le caché par la dissection, pour convaincre d'erreur les adversaires. Du reste, le méthodisme n’est pas très éloigné non plus de l'atomisme d’Épicure. Dès lors, le couplet adressé aux «jeunes philosophes » pourrait concerner les deux groupes de philosophes, opposant ainsi

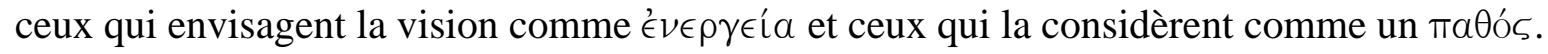

C’est également contre les épicuriens que s'emporte, sensiblement à la même époque que Calcidius, l'auteur des Saturnales, Macrobe, qui défend longuement, lui aussi, l'orthodoxie platonicienne et le rôle de la raison dans la perception sensorielle, ce qu'il résume dans cette formule lapidaire :

\footnotetext{
${ }^{45}$ Avant le c. 246 sur la dissection, nous avons, au chapitre 243, un exemple de pathologie oculaire.

${ }^{46}$ Nemesios von Emesa, Berlin, 1914, p. 26-33.

${ }^{47}$ Cf. De placitis Hippocratis et Platonis, éd. Ph. de Lacy, Berlin, 1980, VII, 5, 22-30.

${ }^{48}$ Ecl. I, 52 [44] = Dox. gr., p. 404.

${ }^{49}$ Cf. L. Edelstein, Ancient Medicine, selected Papers, O. Temkin \& C. L. Temkin édd., Baltimore, 1967, « The Methodists », p. 173-191 [p. 187].
} 
Ergo uidere oculorum est, iudicare rationis ${ }^{50}$.

D’autres auteurs latins ont eux aussi abordé la théorie de la vision de façon plus ou moins développée, que ce soit Apulée, Aulu-Gelle ou Lactance. En dépit de certains échos lexicaux, il est toutefois hasardeux d'en déduire, à la suite de S. Brandt, une source commune qui pourrait être le Tubéron de Varron ${ }^{51}$.

La disparité dans les développements induit sans doute la même disparité dans les sources. Autant les exposés brefs peuvent relever de la tradition doxographique, autant le riche développement de Calcidius - comme celui de Macrobe - dépasse le cadre étroit des résumés scolaires et témoigne d'une originalité indéniable. Par rapport à ses homologues latins, l'exégète platonicien se pose en tout cas en témoin unique de traditions jusqu'ici invérifiables.

Que conclure, au terme de cette étude ? Que les erreurs de traduction, chez Calcidius, n'ont pas d'incidence sur son commentaire, si bien qu'il n'est pas invraisemblable de penser que la traduction ne vient que compléter (de façon erronée !) un commentaire qui est premier. Celui-ci, ne se fondant pas sur une analyse précise du texte, offre une exégèse assez libre, à la fois doxographique et paraphrastique.

Calcidius hérite d'une tradition séculaire dans laquelle le brouillage lexical induit un certain brouillage herméneutique : toutes les théories auxquelles il est fait allusion finissent par se ressembler ! Pourtant, dans ce cadre depuis longtemps figé, l'exégète latin trouve le moyen de prouver son originalité par le double volet - doxographique et exégétique - de son commentaire, et par les témoignages uniques - linguistiques et théoriques - qu'il arrive à offrir.

La théorie de la vision issue du Timée, médiatisée par les doxographes, s’inscrit dans une chaîne qui transmet une tradition très vulgarisée et résumée et qui, par là même, tend à gommer les nuances. Pourtant, la tradition latine des doxographes est plus riche qu'on ne le croit généralement et l'étude de la vision est l'occasion de révéler, dans des contextes différents, un même intérêt pour les sens, et, pour le premier d'entre eux, la vue. C'est également l'occasion de découvrir la tonalité polémique de certains textes latins, et qui, à elle seule, justifierait l'élaboration d'un recueil des Doxographi Latini, sur le modèle des Doxographi Graeci de H. Diels!

\section{Béatrice BAKHOUCHE}

Université Paul Valéry

\footnotetext{
${ }^{50}$ Sat. VII, 14, 17.

51 « Lactantius und Lucretius », NJPhP 143, 1891, p. 225-259, et « Quellen », WSt. 13, 1891, p. 255-292.
} 